\title{
Spectroscopic investigation of carbon migration with tungsten walls in ASDEX Upgrade
}

\author{
A. Kallenbach, R. Dux, J. Harhausen, C.F. Maggi, R. Neu, T. Pütterich, V. Rohde, K. \\ Schmid, E. Wolfrum and the ASDEX Upgrade Team \\ Max-Planck-Institut für Plasmaphysik, IPP-EURATOM Association, \\ D-85748 Garching, Germany
}

\begin{abstract}
Spectroscopic measurements of carbon fluxes in the mainly tungsten-coated ASDEX Upgrade tokamak are analysed with a particle transport and migration code. The transport parameters for deuterium and carbon are calibrated against flux measurements for different experimental conditions. Additional information is obtained from the re-appearance time of carbon after a boronization. The code reproduces the experimental finding that despite a $85 \%$ (2006 campaign) tungsten coverage of the primary PFCs, the carbon concentration in the core and edge plasma is reduced by about a factor 2 only compared to full carbon PFCs. This behaviour is explained by the strong main chamber recycling of carbon in comparison with the loss flux to the inner divertor. The quick recovery of the carbon level in the plasma after a boronisation is explained by carbon influx from the outer divertor.
\end{abstract}

Key words:

PACS: 52.55.Fa, 52.25.Vy, 52.25.Fi, 52.40.Hf

JNM keyword codes: F0400, C0100, M0500, R0900, T0100

PSI-17 keywords: ASDEX Upgrade, First wall, Edge plasma, Impurity sources, Impurity transport

${ }^{*}$ Corresponding author address: Boltzmannstr. 2, D-85748 Garching, Germany 
*Corresponding author email: Arne.Kallenbach@ipp.mpg.de

${ }^{*}$ Corresponding author phone: +49-89-3299 1721

${ }^{*}$ Corresponding author fax: +49-89-3299 1812

\section{Introduction}

ASDEX Upgrade is currently operated with about $85 \%$ of the plasma facing components (PFCs) coated with tungsten. In the main chamber, practically $100 \%$ of the inner wall and about $60 \%$ of the low field side (LFS) limiter surfaces are W-coated. This number even increases when the corresponding $\mathrm{C}$ and $\mathrm{W}$ surface areas are weighted with their impinging D fluxes [1]. Nevertheless, the carbon level in core and divertor plasmas is reduced by only about a factor 2 with the increasing $\mathrm{W}$ coverage over the last years. Figure 1 shows the long term development of the core carbon concentration during a standard H-mode discharge performed routinely in the morning of experimental days [2]. There are considerable variations during an experimental campaign due to boronisation, diagnostic imperfections etc., but a clear trend towards lower carbon concentrations is significant. In Section 2, the present carbon erosion distribution in the main chamber is discussed as derived from spectroscopic influx measurements. A coating-dependent $\mathrm{C}$ sputtering yield is introduced in Section 3 which is used in the particle transport and migration calculations described in Section 4. Several transport parameters appearing in the code calculations are fitted by comparison to steady state experimental data as well as the long term trend with $\mathrm{W}$ coating and the $\mathrm{C}$ recovery phase after a boronisation. 


\section{Carbon influx pattern in the main chamber}

Spatial profiles of CII photon fluxes $(514 \mathrm{~nm})$ are measured along the inner heat shield (HS) and on two LFS limiters [3]. Typical profiles and total numbers of the $\mathrm{C}^{2+}$ influx $(\mathrm{S} / \mathrm{XB}=10)$ extrapolated assuming toroidal symmetry at the HFS and equal fluxes from 12 LFS limiters are shown in figure 2. Despite being fully tungsten coated, the heat shield is the dominant carbon source in the main chamber [4]. The carbon influx from the heat shield is driven by two contributions: Strong recycling at the lower part emerging from chemical erosion in the inner divertor (absent in He plasma) and plasma flux to the upper part caused by radial transport and/or drifts from the LFS. The carbon influx from the upper part of the HS is reduced when the corresponding flux surfaces are limited at the LFS. Practically, strong CII flux from the upper/middle part of the HS is observed when the wall clearance on the HFS mapped to the LFS outer midplane (omp) is smaller than the LFS clearance, corresponding to about less than a factor 2 larger in real space due to the larger HFS flux expansion. The observed carbon sputtering yield from HFS heat shield and LFS limiters depends on the position of the plasma column and the recycling level. This is expected to be caused by an impact energy dependence of the sputtering yield and possibly a flux dependence of $\mathrm{Y}_{\text {chem }}$ at the LFS limiters which leads to reduced yields for flux densities above $\approx 10^{22} \mathrm{~m}^{-2} \mathrm{~s}^{-1}$.

\section{Coating-dependent sputtering yield}

When the coating fraction of a tungsten or boron surface with $\mathrm{C}$ becomes very small, the sputtering yield for $\mathrm{C}$ decreases with the coating fraction principally due to geometrical reasons, since the probability for the projectile to hit a $\mathrm{C}$ atom reduces. When the carbon coating thickness is thinner than the stopping length of the projectile, physical sputtering of 
$\mathrm{C}$ is enhanced in the case of underlying tungsten compared to carbon [5]. The reason is the more efficient back-scattering of the projectile on heavier atoms, which results in a higher momentum available directed off the surface to release the $\mathrm{C}$ atoms.

The evolution of the $\mathrm{C}$ sputtering yield from deposited layers on a tungsten surface as a function of the deposited C areal density was calculated with the codes TRIDYN and DIFFYCHEM [6]. Assuming a $\mathrm{T}_{e}=10 \mathrm{eV}$ plasma, chemical erosion dominates the erosion. Figure 3 shows the deposition dependent sputtering yield calculated under the assumption of $\mathrm{Y}_{\text {chem }}=$ $1 \%$ for a pure $\mathrm{C}$ surface.

We use the following simplified offset-linear ansatz for the observed carbon sputtering yield $Y_{f C}$ for C deposited on a different substrate material:

$$
\begin{array}{cl}
Y_{f C=}=\left(d_{C} / d_{C}^{s a t}\right) \times Y_{C}^{s a t} & d_{C}<d_{C}^{s a t} \\
Y_{f C=Y_{C}^{s a t}} & d_{C} \geq d_{C}^{s a t}
\end{array}
$$

$d_{C}$ is the deposited $\mathrm{C}$ areal density in terms of monolayers and $d_{C}^{\text {sat }}$ the approximated number of monolayers where the linear thickness dependence turns into the thickness independent yield $Y_{C}^{s a t} \cdot d_{C}^{s a t}$ depends on the mean free paths of the projectile and the sputtered atom or molecule in the coating layer as well as on the mass of the substrate atoms. We use $d_{C}^{s a t}=2$ in the following according to the calculations shown in figure 3 .

In a stationary experimental situation without layer growth (erosion dominated PFCs), the impinging carbon flux $\Gamma_{C}=c_{C} \times \Gamma_{D}$ and the eroded flux $Y_{f C} \times \Gamma_{D}$ are equal. $c_{C}$ is the carbon flux fraction towards the surface. The relative equilibrium coating fraction is

$$
d_{C} / d_{C}^{s a t}=c_{C} / Y_{C}^{s a t}, \text { for } c_{C}<Y_{C}^{s a t}
$$

While $Y_{C}^{\text {sat }}$ determines the carbon concentration in the plasma, $d_{C}^{\text {sat }}$ corresponds to the amount 
of carbon at the surface and sets the time constant under which a stationary carbon level develops. To determine this number, a self-consistent modelling of fluxes, sources and sinks is required, which is achieved by the particle migration code described in the next section.

\section{Particle transport calculations}

A numerical code has been set up to describe the carbon particle transport for realistic tokamak conditions with predominantly tungsten (boron) coated surfaces. The main purpose of the code is to reproduce the measured deuterium and carbon fluxes in the main chamber and to provide erosion/redeposition rates and the migration pattern. Core plasma particle transport for deuterium and carbon ions (the latter summed over all ionisation stages) is treated in a similar way as in the transport code STRAHL [7]. The radial flux surface label $\rho_{\text {vol }}$ is calculated from the enclosed volume $\mathrm{V}$, which depends on $\rho_{v o l}$ as the volume of a cylinder with length $2 \pi \mathrm{R}_{\text {axis }}$. Transport coefficients $\mathrm{D}\left(\rho_{v o l}\right)$ and $\mathrm{v}\left(\rho_{v o l}\right)$ are set according to the experience of STRAHL modelling of AUG discharges. Inner and outer SOL are treated as discrete volumes staggered between separatrix and wall. Perpendicular transport in the SOL is described by a diffusion coefficient of $2 \mathrm{~m}^{2} / \mathrm{s}$ and an outward drift velocity of several m/s based on edge profile measurements. Parallel transport from inner and outer SOL to the respective divertors and an ad-hoc drift from the LFS to the HFS scape-off layer are introduced as simple loss times related to Mach numbers with values around 0.5 [8]. Experimental knowledge from post campaign surface analysis is introduced by adjusting the transport parameters in a way that the outer divertor is always a net particle source and the inner divertor the only particle sink and deposition zone.

A non-negligible fraction of particle erosion and transport is related to the occurrence of 
ELMs. ELMs produce bursts of power and particle fluxes at the wall, leading to erosion, and expel particles accumulated around the pedestal top, improving the plasma purity. In the particle transport model, the diffusion coefficient in the edge transport barrier region and, with a smaller factor, in the SOL are repetitively enhanced to simulate ELMs. The main effect of the ELMs is to flush impurities and deuterium ions out of the pedestal region. Due to the weak energy dependence of its sputtering yield, gross carbon release is dominated by the inter-ELM erosion, as observed by fast erosion measurements [3].

In the model calculations, the deuterium gas puff is fed into the LFS scrape-off layer. The amount of gas puff in the calculations is adjusted so as to be equal to the pumped flux derived from ionisation gauge measurements in the pump chamber and the known pumping speed [9]. Only conditions are taken into account where the pumped flux is close to the puffed flux, corresponding to small net wall pumping or gas release. For a typical experimental situation, a deuterium atom recycles about 3-5 times before it is pumped. Since carbon is entrained in the deuterium flux, this recycling pattern is mainly responsible for the build-up of carbon coating fractions on the tungsten (boron) surfaces. A specified net carbon erosion flux in the outer divertor is fed to the upstream separatrix in the model.

\section{Modelling of experimental data}

Two discharge types in AUG have been modelled in detail. The first is the high density phase of the standard H-mode introduced in section 1. Not taking into account effects due to a boronisation, carbon concentrations and fluxes were calculated for different $\mathrm{W}$ coating fractions of inner and outer limiters. Sputtering yields for the HFS are taken according to figure 3, for the LFS half of that value is used to match the experiment. The results are shown 
in figure $4 \mathrm{a}$. The trend of $\mathrm{C}$ reduction compares well with figure 1 if a net eroded carbon influx of $\approx 10^{19}$ atoms/s from the outer divertor is assumed. The modelling reproduces nicely the observation that $\mathrm{W}$ coating of the inner heat shield, where $2 / 3$ of the main chamber recycling occurs, only slightly decreases the carbon core concentration.

Information about the net divertor carbon source can be obtained from the behaviour after a boronisation. On the first experimental day after a boronization, the main chamber carbon and tungsten PFCs are coated with a boron layer $\left(\approx 1 \cdot 10^{22} \mathrm{~B}\right.$ at $/ \mathrm{m}^{2}$ or $75 \mathrm{~nm}$ [10]). Its lifetime is estimated for typical impinging deuterium flux densities (LFS limiter: $2 \cdot 10^{22} \mathrm{~m}^{-2} \mathrm{~s}^{-1}$, HFS heat shield: $3 \cdot 10^{21} \mathrm{~m}^{-2} \mathrm{~s}^{-1}$ ). Assuming a B sputtering yield of 0.3 $\%, 170 \mathrm{~s}$ are obtained for the LFS limiter and $1100 \mathrm{~s}$ for the HFS heat shield, or 24 and 160 typical discharges, correspondingly. These global numbers tend to be reduced by local enhancements of the influx and prolonged by B redeposition.

The recovery of the carbon level in the first pulses after a boronisation is predominantly attributed to carbon influx out of the divertor which is the only PFC with a carbon surface left, since its lower parts are not reached by the boronisation glow discharge. Figure $4 \mathrm{~b}$ shows C fluxes and concentrations for 8 consecutive identical discharges after a boronisation. The 3 discharges prior to this series (thin symbols) had slightly different parameters. The initial $\mathrm{C}$ concentration at the surface is assumed to be $5 \%$ to take into account carbon embedded in the boron layers. The LFS limiter exhibits a quicker timescale in modelling as well as in the measurements due to the higher flux densities on the small surface area. 


\section{Discussion and conclusions}

The temporal variation of the carbon content in ASDEX Upgrade with increasing tungsten PFC coating fraction and after a boronisation has been modelled with a particle transport and migration code. Key ingredients are the strong main chamber recycling and the'geometrical' reduction of the carbon sputtering yield for thin $\mathrm{C}$ layers. The calculations show, in accordance with experimental observations, that due to its recycling nature a major fraction of the primary carbon sources has to be removed before a significant reduction of the core carbon concentration can be observed. The carbon concentration in the plasma is closely coupled to the relative surface coating fraction. For its absolute value, divertor carbon sources are important, which can have a significant contribution to the core $\mathrm{C}$ content in particular for hot divertor conditions [11].

The measured fluxes and yields and derived coating fractions allow to determine the time constant for significant variations of the surface carbon coating. Defining the erosion of 1 monolayer of carbon $\left(0.5 d_{C}^{s a t}\right)$ as a significant variation and neglecting redeposition, for the D flux densities introduced in Sec. 5 and a yield of $0.5 \%$, time scales of $1.5 \mathrm{~s}$ for the HFS and $0.2 \mathrm{~s}$ for the LFS are obtained. Model calculations including the effect of redeposition give much longer time scales of $20 \mathrm{~s}$ and $1 \mathrm{~s}$, respectively. Inspection of the temporal behaviour of fluxes and concentrations reveal, that the outer limiter $\mathrm{C}$ coating and the plasma concentration are closely coupled, while the large HS reservoir (about 30 times the plasma C content per monolayer) lags behind.

The migration modelling calculations also allow to estimate campaign-integrated migration fluxes. We take the conditions of \# 21411 (Fig. 4b) as campaign-typical and assume a full-W inner wall and $25 \%$ C-coverage of the LFS limiters with no boron coating. For $10000 \mathrm{~s}$ 
plasma time, we obtain a net $\mathrm{C}$ erosion in the outer divertor of $3 \mathrm{~g}$, a net erosion at the LFS limiters of $1.5 \mathrm{~g}$ and a deposition of $4.5 \mathrm{~g}$ in the inner divertor. The gross recycling fluxes of $\mathrm{C}$ are larger due to $\mathrm{C}$ redeposition, namely e. $\mathrm{g} .5 \mathrm{~g}$ at the heat shield plus $4 \mathrm{~g}$ backflow from the inner divertor.

The numbers of the migration model based on spectroscopic data are smaller than those obtained from post mortem surface analysis of a previous campaign. For the 2002/2003 campaign, $27 \mathrm{~g}(\mathrm{C}+\mathrm{B})$ have been found deposited in the inner divertor [12]. Possible reasons for the lower carbon deposition derived in this study are the higher tungsten coating fraction (85 vs. $35 \%$ ), the contribution of off-normal erosion events, leading edge erosion and the contribution of a direct carbon drift from the outer to the inner divertor.

Due to the high number of free/fitting parameters (transport coefficients, Mach numbers) and simplifications (limiter geometry, divertor physics) involved, the derived carbon migration pattern still has significant uncertainties. The simplified sputtering yield used does not take into account surface roughness and complicating molecular effects on the chemical erosion dynamics in a mixed $\mathrm{C} / \mathrm{B} / \mathrm{D} / \mathrm{W}$ environment. Possible localised sources due to arc traces or leading edges have not been taken into account. A coupling and combined refinement of $2 \mathrm{D}$ and $3 \mathrm{D}$ edge codes and surface models will be required to remove the mentioned uncertainties.

The planned ITER PFC material choice is quite similar to the present AUG conditions, with carbon mainly left at the strike point zones in the divertor. The observed migration of carbon out of the divertor and through the main chamber in AUG calls for further predictive work for ITER to asses the concomitant tritium co-deposition. 


\section{References}

[1] A. Kallenbach, R. Neu, R. Dux, H.-U. Fahrbach, J. C. Fuchs, et al., Plasma Phys. Controlled Fusion 47 (2005) B207.

[2] F. Ryter, H.-U. Fahrbach, A. Gude, R. Neu, V. Rohde, et al., Plasma Phys. Controlled Fusion 44 (2002) A407.

[3] R. Dux et al., 'Tungsten erosion at the ICRH limiters in ASDEX Upgrade', O-32, this conference, to be published in Journal of Nuclear Materials .

[4] T. Pütterich, R. Dux, J. Gafert, A. Kallenbach, R. Neu, et al., Plasma Phys. Controlled Fusion 45 (2003) 1873.

[5] H. Maier, K. Schmid, and W. Eckstein, J. Nucl. Mater. 337-339 (2005) 480.

[6] K. Schmid and J. Roth, J. Nucl. Mater. 313-316 (2003) 302 .

[7] R. Dux, A. G. Peeters, A. Gude, A. Kallenbach, R. Neu, et al., Nucl. Fusion 39 (1999) 1509.

[8] H. Müller et al., 'Deuterium flow in the scrape-off layer of ASDEX Upgrade', P3-15, this conference, to be published in Journal of Nuclear Materials .

[9] A. Kallenbach, Y. Andrew, M. Beurskens, et al., Plasma Phys. Controlled Fusion 46 (2004) 431.

[10] V. Rohde et al., 'Wall conditioning in ASDEX Upgrade', P2-74, this conference, to be published in Journal of Nuclear Materials .

[11] J. D. Strachan, G. Corrigan, A. Kallenbach, G. F. Matthews, H. Meister, et al., Nucl. Fusion 44 (2004) 772.

[12] M. Mayer, V. Rohde, J. Likonen, E. Vainonen-Ahlgren, K. Krieger, et al., J. Nucl. Mater. 337-339 (2005) 119. 


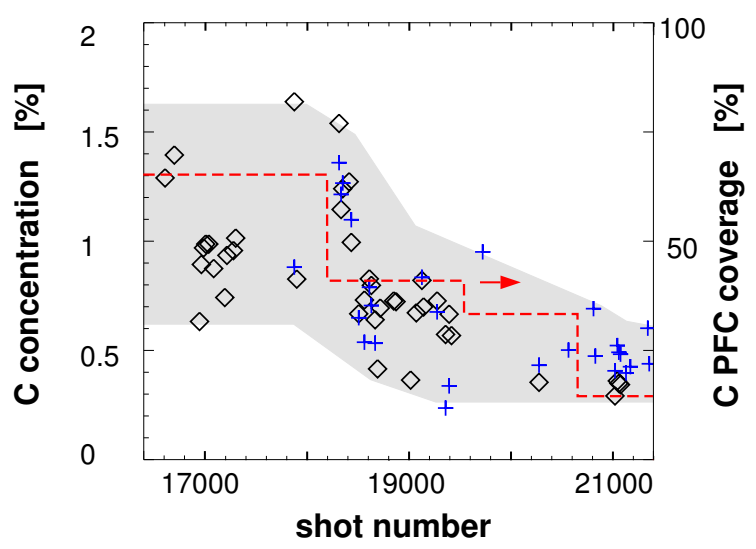

Fig. 1. Long term development of the carbon concentration in the outer core plasma during a high density standard H-mode phase as measured by CXRS with NBI injector 1 (diamonds) and 2 (crosses). The dashed lines indicate the fraction of PFC carbon coverage.
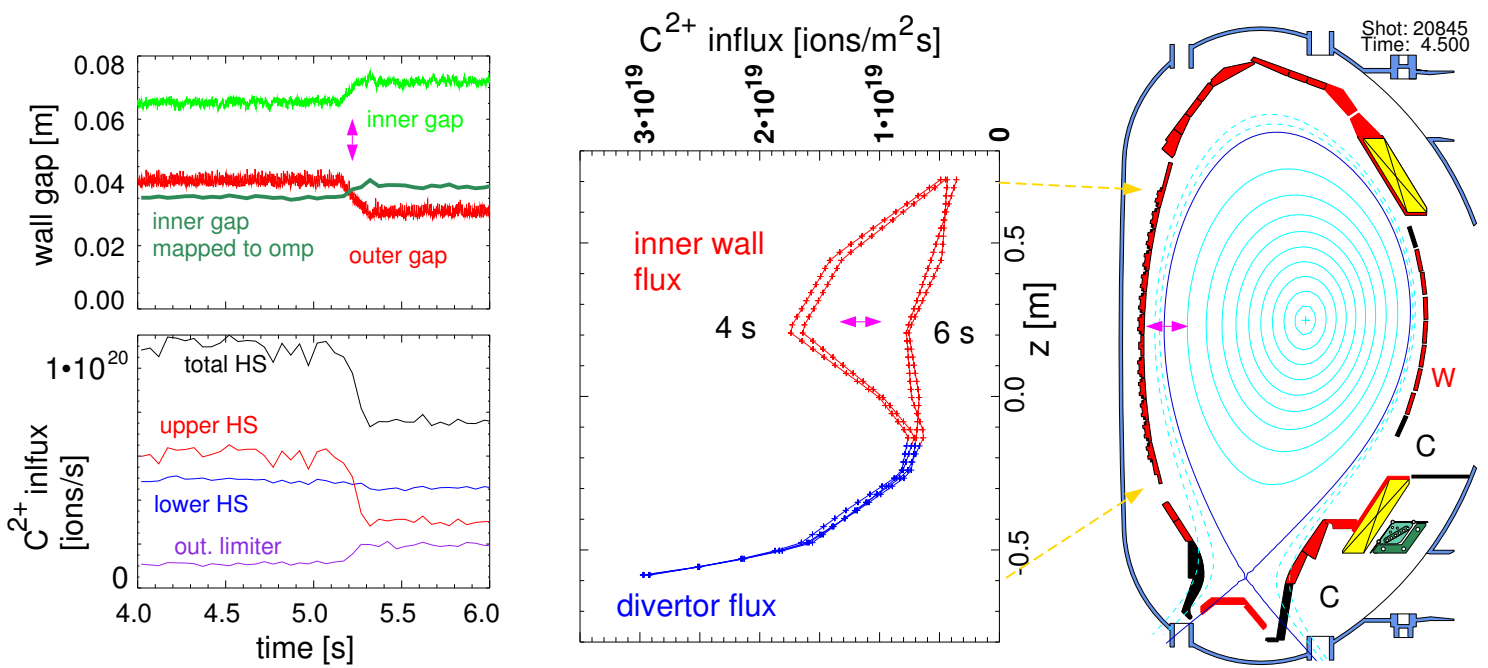

Fig. 2. Spectroscopic measurements of main chamber carbon influx for H-mode conditions and variation of the wall gap (CII line, $514 \mathrm{~nm}, \mathrm{~S} / \mathrm{XB}=10)$ ). The cross section of AUG indicates wall regions with $\mathrm{C}$ and $\mathrm{W}$ coated surfaces. Fluxes are concentrated on limiter surfaces of $9 \mathrm{~m}^{2}$ at the HFS and about $0.4 \mathrm{~m}^{2}$ at the LFS. 


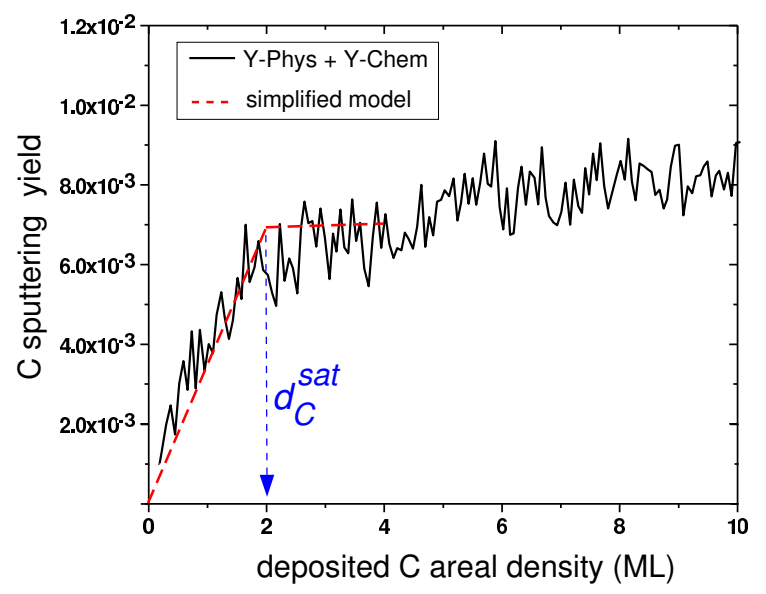

Fig. 3. Monte Carlo calculation of the $\mathrm{C}$ surface coverage dependent yield for $\mathrm{C}$ on tungsten. One monolayer (ML) corresponds to $2 \cdot 2 \cdot 10^{19} \mathrm{C}$ atoms $/ \mathrm{m}^{2}$.
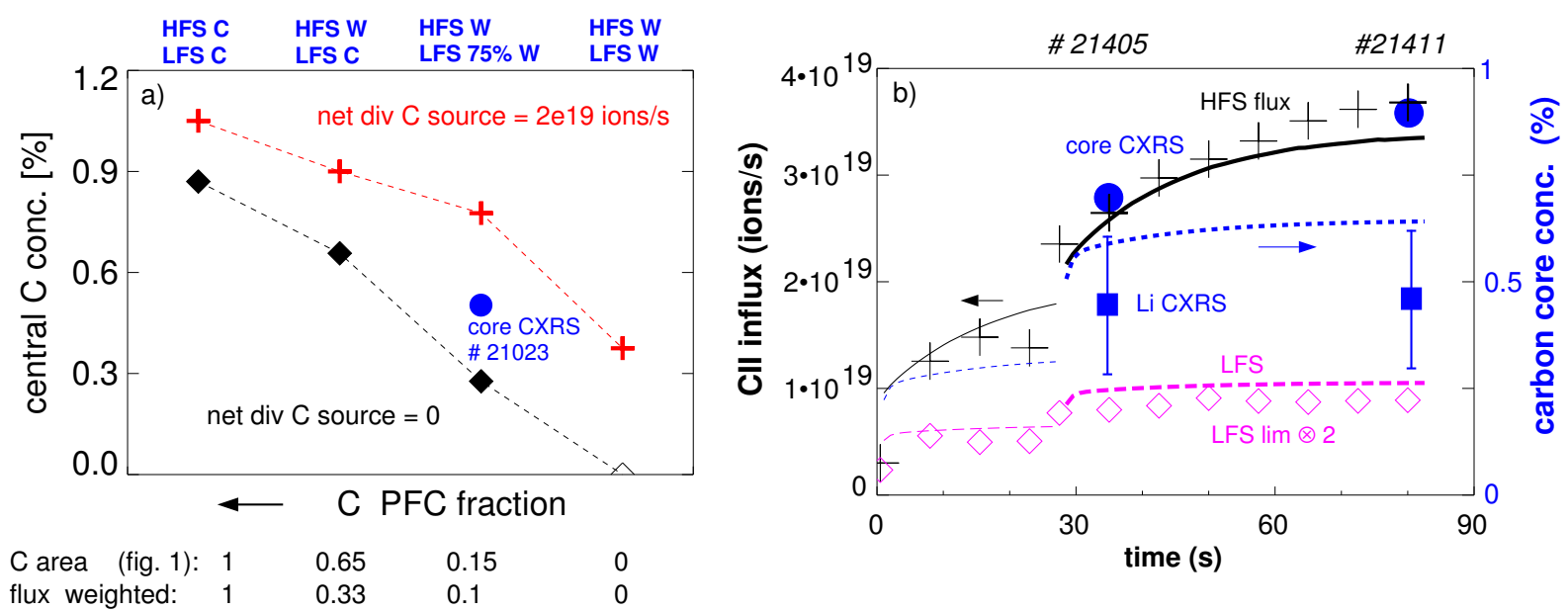

Fig. 4. a) Modelling of the C concentration and HFS and LFS fluxes with changing W coating in AUG with and without net $\mathrm{C}$ ion influx from the divertor. Conditions correspond to Fig. 1. b) Recovery of the carbon level in AUG after a boronisation assuming a $95 \% \mathrm{~B}$ surface coverage and a divertor carbon source of $1.5 \cdot 10^{19}$ atoms/s. Diamonds and crosses show influx measurements from 12 consecutive (last 8 identical) discharges, while circles and squares give bulk core and pedestal carbon concentrations from CXRS measurements. The time denotes integrated flattop time. Lines show the time behaviour of fluxes (black solid and magenta long-dashed) and core concentrations (blue short dashed) calculated with the migration model. 